\title{
Polymorphous Adenocarcinoma (PAC)
}

\author{
Humayun Kaleem Siddiqui ${ }^{1}$ \\ Ausaf Ahmed Khan ${ }^{2}$ \\ Sharjeel Bashir ${ }^{3}$ \\ Syed Muratza Raza Kazmi ${ }^{4}$
}

\author{
BDS, FCPS \\ MBBS, FCPS \\ BDS \\ BDS, FCPS
}

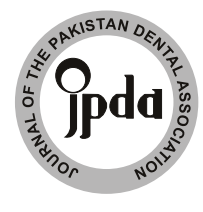

ABSTRACT: Polymorphous adenocarcinoma (PAC) is almost entirely related to minor salivary glands. It is mostly found in the palatal region but on rare it was also observed in other intraoral areas. In recent studies it is observe that there is a potential for histological transformation of the lesion from low to high grade malignancy.

CASE REPORT: A 52 year old male reported to Memon Medical Institute Hospital dental OPD with growth on hard plate for 6 months and mobility of teeth. The lesion was painless with no signs of ocular involvement. Lesion was extending from premaxillary region covering whole of the hard palate to soft palate and beyond. CT scan showed heterogenous enhancing mass with calcifications probably arising from alveolar process of left upper jaw causing complete destruction of left alveolar processes with maxillary bone and hard palate. A plan for total maxillectomy was discussed with the patient and the defect to be filled with a hollow acrylic obturator. On 2 year follow-up, the patient was doning well with no signs of recurrence.

KEY WORDS: Adenocarcinoma, Palate, Salivary glands, Tumour.

HOW TO CITE: Siddiqui HK, Khan AA, Bashir S, Kazmi SMR. Polymorphous adenocarcinoma (PAC). J Pak Dent Assoc 2018;27(3):152-56.

DOI: https://doi.org/10.25301/JPDA.273.152

Received: 05 October 2017, Accepted: 15 February 2018

\section{INTRODUCTION}

$\mathrm{P}$ olymorphous adenocarcinoma (PAC) is almost entirely related to minor salivary glands. ${ }^{1-3}$ In 1983 , Freedman et al, described and named it lobular carcinoma on its resemblance to lobular carcinoma of the breast. ${ }^{2,4}$ On histopathology it is frequently misdiagnosed as pleomorphic adenomas, monomorphic adenomas, adenoid cystic carcinoma, malignant pleomorphic adenomas or adenocarcinoma. ${ }^{4,5}$

In 1984, Batsakis et al, introduced the term Polymorphous low grade adenocarcinoma (PLGA). ${ }^{6}$ This carcinoma is mostly found in the palatal region but on rare it was also observed in other intraoral areas like upper lip, buccal mucosa, posterior tongue and retromolar trigone. ${ }^{5,7}$ It is usually found in 6th to 7 th decade of life and occasioinally in adolescents. ${ }^{8-10}$ It is the second most common malignant tumor of the minor salivary gland followed by mucoepidermoid carcinoma. ${ }^{7} \mathrm{PAC}$ is extremely infrequent

1. Associate Professor, Oral and Maxillofacial Surgery Head of Oral and Maxillofacail Surgery Dept Baqai Dental College, Baqai Medical Univeristy Karachi, Pakistan.

2. Consultant, ENT Surgeon Memon Medical Institute Hospital Karachi, Pakistan.

3. Masters Candidate, Oral and Maxillofacial Surgery University of Karachi Karachi, Pakistan.

4. Professor, Prosthodontics Fatima Jinnah Dental College Karachi, Pakistan.

Corresponding author: "Dr. Sharjeel Bashir" < drsharjeelbashir@yahoo.com $>$ in the major salivary glands. ${ }^{11}$ Recently detection of PAC has been reported in other areas instead of minor salivary glands like major salivary glands. ${ }^{12}$ The breas ${ }^{13}$, paranasal sinuses ${ }^{14}$, the skin and orbit ${ }^{15}$, vulva and vagina ${ }^{16}$, Lung lesions were also reported both as metastatic and primary lesions. ${ }^{8,17}$ Possible variant of PAC is Cribriform Adenocarcinoma of Tongue (CAT) and other Salivary Glands (CASG), but it is not clear yet whether is shows genuine entity or just unusual growth pattern in PAC. ${ }^{18}$ Histological and molecular features overlap between PAC and CASG, but CASG is a distinct tumor entity and differ from PAC by location, behavior, cytology and histologically. ${ }^{19}$

Histological and immunohistochemical features of these lesions are similar to the minor salivary gland lesions. ${ }^{3,12,13,16}$ PAC is clinically innocuous in-spite of its local infiltrative pattern and perineural and pervascular invasive features. ${ }^{5,20}$ Less than $1 \%$ of cases shows low grade malignant potential ${ }^{9,20}$ And distant spread is rare. In recent studies it has been shown that the lesion is locally aggressive in few of cases. ${ }^{10,20,21}$ Aggressive clinical progression have been associated with various factors like inadequate excision with positive margin, repeated surgery, exposure to radiation, prolonged duration and occurrence in a young patient. ${ }^{10,20-22}$ 
On histopathology PAC is non-encapsulated and shows infiltrative borders. Growth pattern shows high variability that include tubular, solid, papillary, microcytic, cribriform, fascicular, single file and strand like arrangement. ${ }^{20,21}$ This morphologically different growth patterns are responsible for difficulty in diagnosis and confusion with other salivary gland tumors like pleomorphic adenoma and adenoid cystic carcinoma. In these types of cases immunohistochemical studies are indicated. ${ }^{23}$

In recent studies a potential for histological transformation of the lesion from low to high grade malignancy was observed ${ }^{10,20}$ and the papillary cystic pattern was associated with aggressive clinical behavior. ${ }^{5,20-22}$

\section{CASE REPORT}

A 52 year old male reported to Memon Medical Institute Hospital dental OPD with growth on hard palate for 6 months and mobility of teeth. A previous biopsy showed ameloblastoma at another hospital. He was generally healthy otherwise. Extraorally the lesion was painless with no signs of ocular involvement. Paraesthesia in the division of infraorbital region was not found. All the ocular movements were in normal range with intact vision on both sides. Submandibular lymph nodes were not palpable. Intraorally a $4 \times 3 \mathrm{~cm}$ swelling was noted with ulceration in the middle palate as a result of previous biopsy. The lesion was extending from premaxillary region covering whole of the hard palate to soft palate and beyond. The overlying mucosa was swollen with intact predominantly pink mucosal surface. Slight mobility of the maxillary teeth was noted. On palpation the lesion was firm to soft. There was no active discharge or bleeding. Left buccal and labial sulcus was slightly enlarged whereas right sulcus was in normal limits.

CT scan axial images showed heterogenous enhancing soft tissue mass with amorphous calcification, measuring $7.9 \times 9.2 \times 7.6 \mathrm{~cm}$ (CC x AP x Transverse), arising from alveolar processes of left upper jaw causing complete destruction of left alveolar process with maxillary bone and hard plate. The Mass was extending and occupying left maxillary sinus, nasopharynx and posterior aspect of both nasal cavities. It was extending from left cheek crossing the midline and reaching up to the medial wall of right maxillary sinus and superiorly extending into the ethmoidal sinuses. Fluid density and air was seen in right maxillary sinus could be due to obstruction by mass resulting in sinusitis. Expansion of posterior nasal cavity and nasopharynx was also noted. Mass was also abutting with both medial pterygoid plates. No abnormality was seen in the prelaryngeal space, valleculae and pyriform sinuses. The laryngeal, arytenoids and cricoids cartilages were normal. The thyroid gland showed homogenous enhancement with no focal mass. Major blood vessels of neck and both vocal cords were normal. Few subcentimeter lymphnodes were seen at submental, submandibular and right supraclavicular levels.

A plan for total maxillectomy was discussed with the patient and the defect to be filled with a hollow acrylic obturator. Under GA a Weber-Fergusson incision with infraorbital extension on left side was used to expose the tumour. A combination of sharp and blunt dissection with judicious use of periosteal elevator was used to excise the tumour with a margin of $1.5 \mathrm{~cm}$ overall. The tumour was removed along with the normal tissues from nasal cavity, paranasal sinuses, inferior turbinate and up to the anterior pharyngeal wall a thin sling of soft palate tissue was maintained to aid in closure and help in retention of obturator. A thin shelf of right maxillary antral wall was left with two teeth to aid in retention of the final denture. After tumour excision, a split thickness skin graft was applied to the large cavity followed by Antibiotic impregnated ribbon gauze placement and a prefabricated initial obturator was placed with circumzygomatic wires bilaterally.
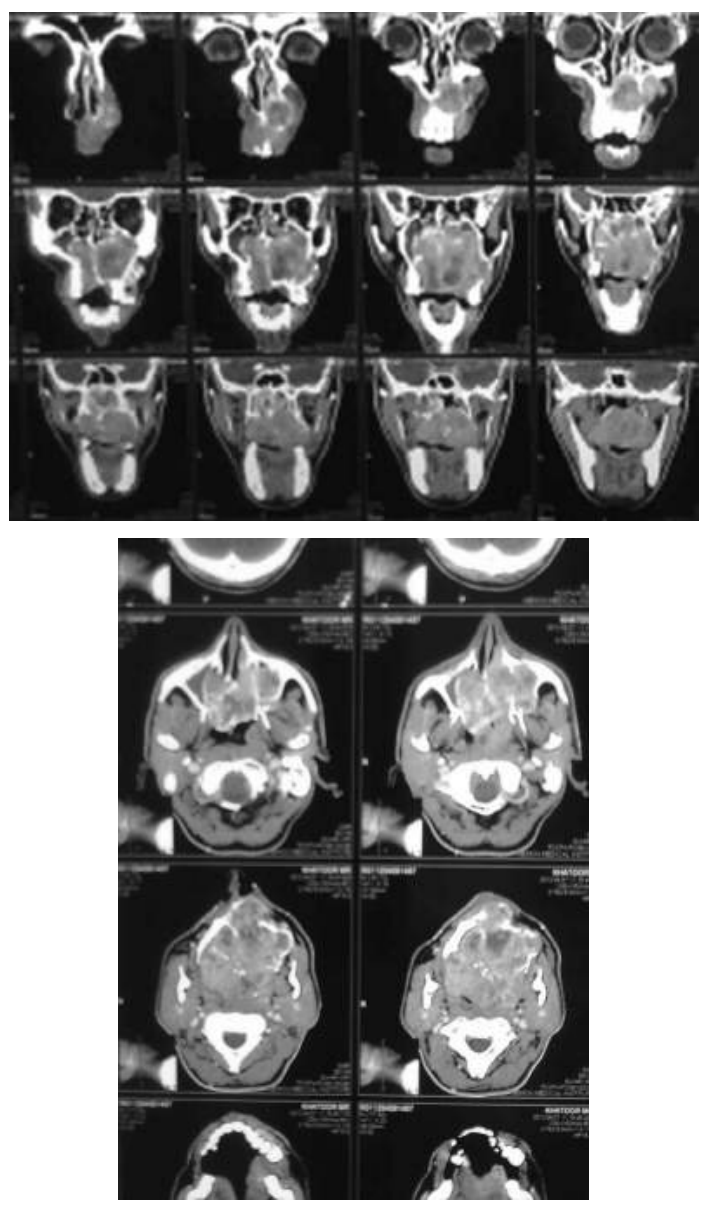

Figure 1A \& 1B: Pre-operative Coronal and Axial section on CT Scan 


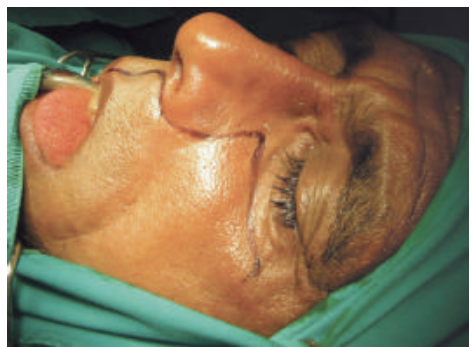

Figure 1: Weber-Fergusson incision given by using number 15 surgical blade to raise flap.

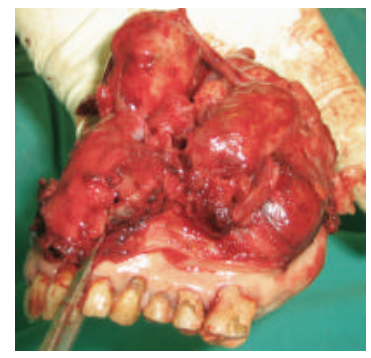

Figure 2D: Another view of Gross specimen showing complete removed tumor mass along with maxillary teeth.

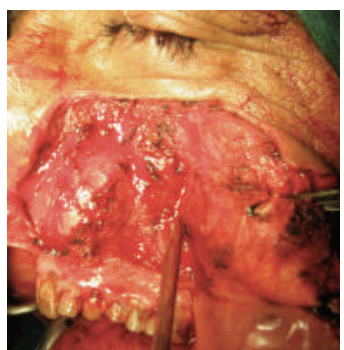

Figure 2A: After raising the flap for removal of the pathology, shows tumor mass.

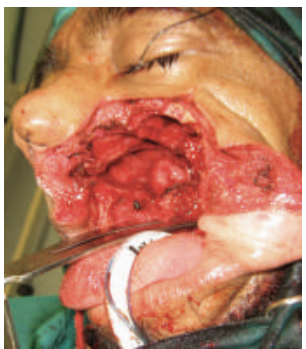

Figure 2B: After Sub-Total maxillectomy, tumor completely removed showing inner lining of the nasal cavity.

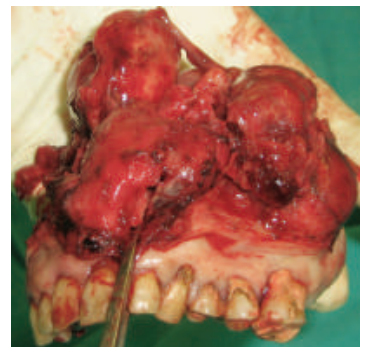

Figure 2C: Gross specimen showing the removed tumor mass along with 10 maxillary teeth, left maxillary first molar to right maxillary first premolar

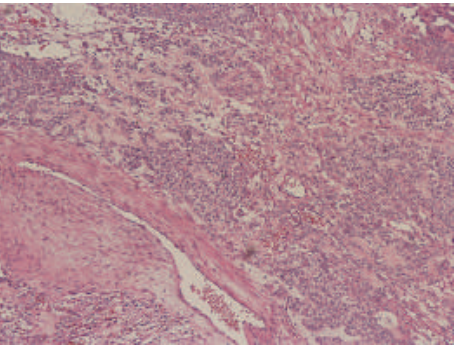

Figure 3A: A 10x photomicrograph ( haematoxylin \& eosin) showing nest of neoplastic cell arranged in fused glands along with few areas showing cribriforming pattern of neoplastic cell.

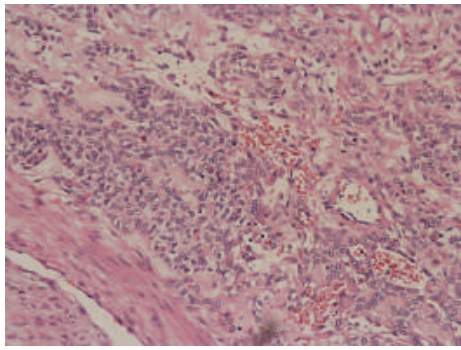

Figure 3A: (A 40x photomicrograph ( haematoxylin \& eosin) showing the characteristic nuclear feature. cribriform areasshowed plump columnar cells with moderate atypia.)
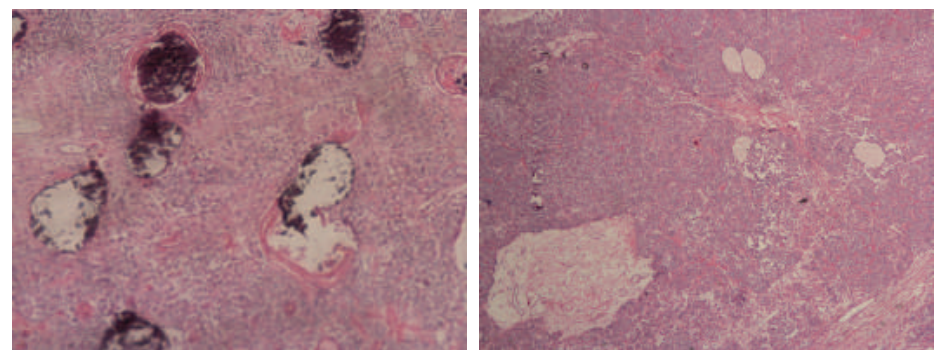

Figure 3B: A photomicrograph (haematoxylin \& eosin)

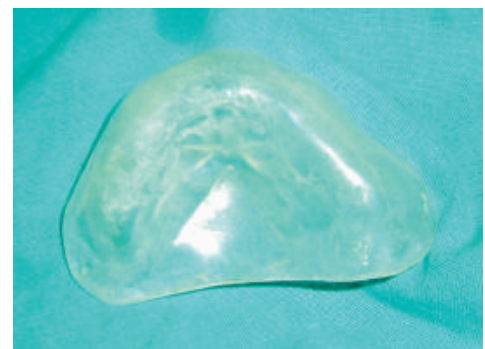

Figure 4: Showing initial obturator plate that is fixed with circumzygomatic wiring to occlude the defect

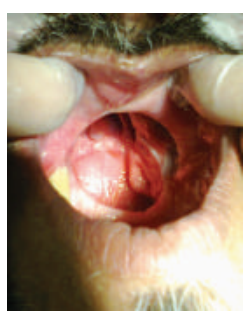

Figure 5C: Follow up picture taken after 1 year showing healthy mucosa, clinically no evidence of recurrence.
Figure 5B: Intraoral view of the denture along with obturator showing completely closed the defect from where tumor was removed 
An intraoperated biopsy was sent for further confirmation. Sections examined revealed an invasive lesion composed of nest of neoplastic cell arranged in fused glands along with few areas showed cribriforming pattern of neoplastic cells. These nest and cribriform areas showed plump columnar cells with moderate atypia. At places plasmacytoid appearance of cells was also noted.

The patient was followed after two weeks with interim obturator and final obturator with maxillary teeth with the help of maxillofacial prosthodontist. On a two year follow up the patient was doing well with no signs of recurrence.

\section{DISCUSSION}

Polymorphous Adenocarcinoma (PAC) is an uncommon tumour and mostly related to minor salivary glands. ${ }^{1,3}$ It was first described in 1983 by Freedman et al, and it was named lobular carcinoma on its resemblance to lobular breast carcinoma. ${ }^{2,4}$ In 1984, Batsakis et al, introduced the term Polymorphous low grade adenocarcinoma (PLGA). ${ }^{6}$ The most preferred location for this tumour is noted to be the palatal region and minor salivary glands are involved in majority of cases ${ }^{3}$ but on rare occasion it was documented in other intra oral areas also like upper lip, buccal mucosa, posterior tongue and retromolar trigone. ${ }^{5,7}$ Patients mean age with PAC is noted to be approximately 58 years, and male to female ratio is around $1: 2 .{ }^{24} \mathrm{~A}$ study of One-sixtyfour patients of PAC with average follow up nearly 10 years, showed $97.6 \%$ of all patients treated by surgery were either alive or died without any evidence of tumour recurrence. ${ }^{24}$ From 164 patients, only 4 had evidence of tumour recurrence at the last follow up. Three died and only one was alive with a tumour. ${ }^{11}$

We describe here a case of PAC arising from minor salivary gland of the palate. The lesion was arising from alveolar process of left upper jaw causing complete destruction of left alveolar process, extending and occupying left maxillary sinus, nasopharynx and posterior aspect of both nasal cavities. From left cheek crossing the midline and reaching up to the medial wall of right maxillary sinus and superiorly extending into the ethmoidal sinuses. Expansion of posterior nasal cavity and nasopharynx was also noted. The Mass was also abutting with both medial pterigoid plates, no evidence of metastatic disease was found.

PAC is almost entirely arising from minor salivary glands and is considered to be a low grade. ${ }^{11}$ This tumours is generally slow growing. ${ }^{11}$ Previously some tumours have been reported to have variable pattern of local recurrence and metastasis. ${ }^{11}$ The extent and radiographic features of PAC were described in very few cases. ${ }^{11}$ De magalhaes et al, ${ }^{25}$ document a mandibular lesion with unusual radiographic features that resemble to a benign tumour. ${ }^{11}$ On the basis of these findings, an ameloblastoma was considered to be preferred diagnosis in that case. An intraosseous PAC of the maxilla is described by Sato et al, in this a panoramic radiographic features were more similar to a radicular cyst, but in C.T scan irregular scalloping and perforation of the cortical borders indicates a malignant lesion. ${ }^{11}$

Our case showed different radiographic features that resembled to slow growing tumour with aggressive. On C.T scan a heterogenous soft tissue mass with amorphous calcification was seen that was arising from alveolar processes of left upper jaw causing complete destruction of left alveolar processes with maxillary bone and hard palate. Mass was extending from left vestibule crossing the midline and reaching upto the medial wall of right maxillary sinus and superiorly extending into the ethmoidal sinuses. Expansion of posterior nasal cavity and nasopharynx was noted. Few sub-centimeter lymphnodes was seen at submental, submandibular and right supraclavicular levels. These all features were resembling to a low grade tumour of minor salivary gland origin. ${ }^{11}$ At some occasion these tumours could be misdiagnosed due to its varied histomorphological patterns. ${ }^{11}$

Histopathological examination of excised tumour revealed an invasive lesion composed of nest of neoplastic cell arranged in fused glands along with few areas showing cribriforming pattern of neoplastic cell. These nest and cribriform areas showed plump columnar cells with moderate atypia. At places plasmacytoid appearance of cells are also noted. Based on morphology and PAC.

In most of cases of PAC excision of the primary lesion with wide margin is recommended, but in some cases modified radical neck dissection is indicated. Recurrences may occur after a long period, so for prevention prolonged and systemic follow up is recommended. ${ }^{22,24,26}$ In majority of cases prognosis is good but mortality rate up to $12.5 \%$ was documented in a research comprising of 40 patients that are followed up for the time of ten years. ${ }^{21}$ Because of this behavior some authors suggested to remove the term "low grade" from the name. ${ }^{27}$

\section{CONFLICT OF INTEREST}

None declared.

\section{REFERENCES}

1. Soames JV, Southam JC. Diseases of Salivary Glands. Oral Pathology. 4th ed. 2005: 218. 
2. Olusanya AA, Akadiri OA, Akinmoladun VI, et al. Polymorphous low grade adenocarcinoma: literature review and report of lower lip lesion with suspected lung metastasis. J maxillofac Oral Surg. 2011; 10(1): 60-63.

3. Hannen EJ, Bulten J, Festen J, et al. Polymorphous low-grade adenocarcinoma with distant metastases and deletions on chromosome 6q23-qter and 11q23-qter: a case report. J ClinPathol. 2000; 53: 942-945. https://doi.org/10.1136/jcp.53.12.942

4. Freedman P, Lumerman H. Lobular carcinoma of intraoral minor salivary gland origin: report of twelve cases. Oral Surg Oral Med Oral Pathol 1983; 56: 157-166.

https://doi.org/10.1016/0030-4220(83)90282-7

5. Vincent SD, Hammond HL, Finkelstein MW. Clinical and therapeutic features of polymorphous low grade adenocarcinoma. Oral Surg Oral Med Oral Pathol. 1994; 77: 41-47.

https://doi.org/10.1016/S0030-4220(06)80105-2

6. Evans HL, Batsakis JG. Polymorphous low-grade adenocarcinoma of minor salivary glands. A study of 14 cases of distinctive neoplasm. Cancer. 1984; 53: 935-942.

https://doi.org/10.1002/10970142(19840215)53:4<935::AID-CNCR28205 $30420>3.0 . \mathrm{CO} ; 2-\mathrm{V}$

7. Waldron CA, el-Mofty SK, Gnepp DR. Tumors of the intraoral minor salivary glands: a demographic and histologic study of 426 cases. Oral Surg Oral Med Oral Pathol. 1988; 66: 323-333

https://doi.org/10.1016/0030-4220(88)90240-X

8. Lee VK, McCaughan BC, Scolyer RA. Polymorphous low-grade adenocarcinoma in the lung: a case report. Int J Surg Pathol. 2004; 12: 287-292.

https://doi.org/10.1177/106689690401200313

9. Lengyel E, Somogyi A, Godney M, et al. Polymorphous low-grade adenocarcinoma of the nasopharynx. Case report and review of literature. Strahlenther Onkol. 2000; 176: 40-42.

https://doi.org/10.1007/PL00002304

10. Tanaka F, Wada H, Inui K, et al. Pulmonary metastasis of polymorphous low-grade adenocarcinoma of the minor salivary gland. Thorac Cardiovasc Surg. 1995; 43: 178-180.

https://doi.org/10.1055/s-2007-1013795

11. Potluri A, Prasad J, Levine S, et al. Polymorphous low-grade adenocarcinoma: a case report. Dento-maxillofacRadiol. 2013; 42:480484.

https://doi.org/10.1259/dmfr/14804843

12. Yih WY, Kratochvil FJ, Stewart JC. Intraoral minor salivary gland neoplasms: review of 213 cases. J Oral Maxillofacial Surg. 2005; 63: 805810.

https://doi.org/10.1016/j.joms.2005.02.021

13. Asioli S, Marucci G, Ficarra G, et al. Polymorphous adenocarcinoma of the breast. Report of three cases. Virchows Arch. 2006; 448: 29-34. https://doi.org/10.1007/s00428-005-0084-2

14. Charous DD, Cunnane MF, Rosen MR, et al. Recurrent polymorphous low-grade adenocarcinoma manifesting as a sinonasal mass: a case report.
Ear Nose Throat J. 2005; 84: 354-357.

15. Thomas KM, Cumberworth VL, McEwan J. Orbital and skin metastasis in polymorphous lowgrade adenocarcinoma of the salivary gland. J Laryngol Otol. 1995; 109: 1222-1225.

https://doi.org/10.1017/S0022215100132517

16. Young S, Leon M, Talerman A, et al. Polymorphous low-grade adenocarcinoma of the vulva and vagina: a tumor resembling adenoid cystic carcinoma. Int J Surg Pathol. 2003; 11: 43-49.

https://doi.org/10.1177/106689690301100113

17. Kyu Do Cho, Ji Han Jung, Deog Gon Cho, et al. Primary polymorphous low grade adenocarcinoma of lung treated by sleeve bronchial resection: a case report. J Korean Med Sci. 2007; 22: 373-376.

https://doi.org/10.3346/jkms.2007.22.2.373

18. WHO Classification of Tumours: Pathology and Genetics Head and Neck Tumours, IARC Press, 2005.

19. WHO classification salivary tumours: An update on Histopathology of Salivary Gland Tumors, La Spezia, Italy, Oct 18-20, 2017.

20. Simpson RH, Pereira EM, Ribeiro AC, et al. Polymorphous low grade adenocarcinoma of the salivary glands with transformation to high-grade carcinoma. Histopathology. 2002; 41: 250-259.

https://doi.org/10.1046/j.1365-2559.2002.01439.x

21. Harry L, Evans MD, Mario A, et al. Polymorphous low-grade adenocarcinoma a study of 40 cases with long-term follow up and an evaluation of the importance of papillary areas. Am J Surg Pathol. 2000; 24: 1319-1328.

https://doi.org/10.1097/00000478-200010000-00001

22. Pelkey TJ, Mills SE. Histologic transformation of polymorphous low-grade adenocarcinoma of salivary gland. Am J Clin Pathol. 1999; 111: 785-791.

https://doi.org/10.1093/ajcp/111.6.785

23. Chaâbouni S, Ayadi L, Dhouib H, et al. Polymorphous low-grade adenocarcinoma: a palatine and a labial location. Rev Stomatol Chir Maxillofac. 2008; 109: 178-182.

https://doi.org/10.1016/j.stomax.2008.04.002

24. Castle J, Thompson L, Frommelt RA, Wenig B, Kessler H. Polymorphous low grade adenocarcinoma: a clinicopathologic study of 164 cases. Cancer 1999; 86: 207-219.

https://doi.org/10.1002/(SICI) 10970142(19990715)86:2<207::AID-CNCR 4>3.0.CO;2-Q

25. de Magalhaes M, de Magalhaes R, de Araujo V, de Sousa S. Polymorphous low grade adenocarcinoma presenting an uncommon radiographic aspect. DentomaxillofacRadiol 2006; 35: 209-212. https://doi.org/10.1259/dmfr/17652888

26. Gonzalez-Garcia R, Rodriguez-Campo FJ, et al. Polymorphous lowgrade adenocarcinoma of the palate: report of cases. Auris Nasus Larynx. 2005; 32: 275-280.

https://doi.org/10.1016/j.anl.2005.03.019

27. Speight PM, Barrett AW. Salivary gland tumors. Oral Dis. 2002; 8: 229-240.

https://doi.org/10.1034/j.1601-0825.2002.02870.x 